\title{
Correction to: Familial intellectual disability as a result of a derivative chromosome 22 originating from a balanced translocation $(3 ; 22)$ in a four generation family
}

Kaihui Zhang ${ }^{1+}$, Yan Huang ${ }^{2 \dagger}$, Rui Dong ${ }^{1+}$, Yali Yang ${ }^{2}$, Ying Wang ${ }^{1}$, Haiyan Zhang ${ }^{1}$, Yufeng Zhang ${ }^{1}$, Zhongtao $\mathrm{Gai}^{1 *}$ and Yi Liu ${ }^{1 *}$

\section{Correction}

In the original publication [1] the author names were in the wrong order. The correct version can be found in this Correction. The original article has been updated to rectify this error.

Incorrect version:

Zhang Kaihui, Huang Yan, Dong Rui , Yang Yali, Wang Ying , Zhang Haiyan, Zhang Yufeng, Gai Zhongtao and Liu Yi

Correct version:

Kaihui Zhang, Yan Huang, Rui Dong, Yali Yang, Ying Wang, Haiyan Zhang, Yufeng Zhang, Zhongtao Gai and Yi Liu.

\section{Author details \\ 'Pediatric Research Institute, Qilu Children's Hospital of Shandong University, 23976 Jingshi Road, Jinan 250022, Shandong, China. ${ }^{2}$ Rehabilitation Center, Qilu Children's Hospital of Shandong University, 23976 Jingshi Road, Jinan 250022, Shandong, China.}

Published online: 23 April 2018

\section{Reference}

1. Zhang $\mathrm{K}$, et al. Familial intellectual disability as a result of a derivative chromosome 22 originating from a balanced translocation (3;22) in a four generation family. Mol Cytogenet. 2018;11:18. https://doi.org/10.1186/ s13039-017-0349-x

\footnotetext{
*Correspondence: gaizhongtao@sina.com; y.liu99@yahoo.com

${ }^{\dagger}$ Equal contributors

'Pediatric Research Institute, Qilu Children's Hospital of Shandong University, 23976 Jingshi Road, Jinan 250022, Shandong, China
} 